\title{
Self-Assembled Nanoparticles for Integrated Cancer Imaging and Therapy
}

\author{
Hongwei Duan \\ Department of Biomedical Engineering, Emory University and Georgia Institute of \\ Technology, 101 Woodruff Circle, WMB, Suite2007, Atlanta, GA30322
}

Recent advances in nanotechnology have continued expanding the arsenal of imaging and therapeutic platforms to fight major human diseases, especially for various types of cancer. For cancer research, nanoparticles have been specifically directed to tumor through both passive and active targeting approaches by taking advantaging of the tumorfeatured enhanced permeability and retention (EPR) effect or molecular recognition of targeting ligands (antibody, peptide and small molecules), respectively. Owing to their large surface-to-volume ratio, nanoparticles have sufficient surface areas for conjugation of multiple targeting ligands, imaging and therapeutic agents, offering possibilities to design multi-modality platforms with integrated functionalities. For example, nanoparticles linked with imaging modality, i.e., optical imaging probes and therapeutic agents, for example, anticancer drugs would enable simultaneous tumor imaging and treatment and also would provide new mechanisms to accelerate the process of drug discovery. This talk is focused on a new class of self-assembled polymeric nanoparticles, which exhibit long blood circulation and dynamic structures, for in-vivo cancer imaging and therapy. 